\title{
REGENERATION AND GENERAL MARKOV CHAINS
}

\author{
VLADIMIR V. KALASHNIKOV \\ Institute of Systems Analysis \\ 9, Prospect 60 let Oktyabrya \\ 117312 Moscow, RUSSIA
}

(Received January, 1994; revised May, 1994)

\begin{abstract}
Ergodicity, continuity, finite approximations and rare visits of general Markov chains are investigated. The obtained results permit further quantitative analysis of characteristics, such as, rates of convergence, continuity (measured as a distance between perturbed and non-perturbed characteristics), deviations between Markov chains, accuracy of approximations and bounds on the distribution function of the first visit time to a chosen subset, etc. The underlying techniques use the embedding of the general Markov chain into a wide sense regenerative process with the help of splitting construction.
\end{abstract}

Key words: General Markov Chain, Regenerative Process, Ergodic Theorem, Finite Approximation, Test Function, Rare Event.

AMS (MOS) subject classifications: $60 \mathrm{~J} 05,60 \mathrm{~K} 99$.

\section{Introduction}

In paying tribute to Lajos Takács, one must remember that he is one of the few outstanding mathematicians who paid much attention to transient behavior in queueing systems, which he investigated by analytic methods in his classic book Introduction to the Theory of Queues (Oxford University Press, New York, 1962). The problem of transient behavior turned out to be extremely difficult even for simple queueing models. Because of this, it is often reasonable not to obtain explicit formulas for different characteristics but to approximate these characteristics or to investigate general properties of underlying processes.

This paper deals with general Markov chains. The theory of such chains has undergone dramatic changes recently. These changes were caused by the discovery of an embedded renewal process and, therefore, the feasibility of employing a recurrent events technique (as in Feller [3]) which has been employed successfully for denumerable chains. We refer to Nummelin [13] and Meyn and Tweedie [14] for further details. Though a general Markov chain can be considered as a regenerative process, there are at least two features that distinguish the general case from the denumerable or finite case. First, a general Markov chain (even Harris-recurrent) has no "proper atom" (by Nummelin's terminology, this is an analog of a recurrent state) and so a traditional choice of recurrent times to a specific state as regenerative epochs is inadmissible in general. This was the reason for E. Nummelin to suggest a so-called "splitting," which enables one to construct an enlarged Markov chain comprising the initial chain as a component and possessing a proper 
atom. Second, a regenerative process, describing a general Markov chain, is not a classic sense regenerative process introduced in Smith [16] with independent cycles, but represents a wide sense regenerative process. That class of regenerative processes has been studied intensively in Asmussen [1] and Thorisson [17-19].

Although a representation of a general Markov chain as a regenerative process was initially used for investigating the steady state regime, such a representation can also be used for other purposes. This paper is intended to show how the "regenerative approach" elaborated recently in Kalashnikov [5], [7], [8], and Thorisson [20] can be employed for studying the continuity property, obtaining finite approximations, proving limit theorems, and deriving various bounds for general Markov chains. We will refer known assertions from the theories of regenerative and Markov processes to as "Propositions." They are given here without proofs. Their corollaries concerning Markov chains are called "Lemmas" and "Theorems", and they are furnished with proofs.

\section{Necessary Definitions}

We will preserve definitions introduced in Nummelin [13] and Meyn and Tweedie [14]. Let $\boldsymbol{X}=\left(X_{0}, X_{1}, \ldots\right)$ be a general Markov chain with state space $\left(\boldsymbol{\chi}, \Re_{B}\right)$ that is a complete separable metric space endowed with metric $h$ and $\sigma$-algebra $\Im_{B}$ generated by all open subsets of $\chi$. Denote its initial distribution by $p_{0}(\cdot)$, its $m$-step transition function by $\boldsymbol{P}(x, m ; \cdot)$, and let

$$
\begin{gathered}
\mathbf{P}(x ; \cdot)=\mathbf{P}(x, 1 ; \cdot), \\
\mathbf{P}_{x}(\cdot)=\mathbf{P}\left(\cdot \mid \boldsymbol{X}_{0}=x\right) .
\end{gathered}
$$

Let $\mathfrak{H b}_{+}$denote the collection of $\sigma$-finite nonnegative measures $\varphi$ that are defined on $\mathfrak{B}$ and satisfy the inequality $\varphi(\chi)>0$. The following definitions are standard and can be found in Nummelin [14].

Definition 1: We say that state $x \in \chi$ leads to $B \in \Im_{B}$ and denote this by $x \rightarrow B$, if $\mathbf{P}(x, n ; B)>0$ for some $n>0$.

Definition 2: Given $x \in B$ for any $x \in \chi, B \in \mathfrak{B}$, and $\varphi(B)>0$, Markov chain $\boldsymbol{X}$ is called $\varphi$ irreducible.

Definition 3: Markov chain $\boldsymbol{X}$ is called irreducible if it is $\varphi$-irreducible for at least one measure $\varphi \in \mathfrak{A b}_{+}$.

Proposition 1 (Nummelin [14]): Given an irreducible Markov chain $\boldsymbol{X}$, there exists a maximal irreducibility measure $\Phi \in \mathcal{1}+$ for which

(i) $\boldsymbol{X}$ is $\Phi$-irreducible;

(ii) if $\boldsymbol{X}$ is $\varphi$-irreducible, then $\varphi \ll \Phi$ (this means $\Phi(B)=0 \Rightarrow \varphi(B)=0$, $B \in \mathfrak{B})$;

(iii) if $\Phi(B)=0$ and $B^{\prime}=\{x: x \rightarrow B\}$, then $\Phi\left(B^{\prime}\right)=0$.

Proposition 2 (Nummelin [14]): Given an irreducible Markov chain $\boldsymbol{X}$, there exist a subset $C \in \mathfrak{B}$, an integer $m \geq 1$, a positive $\beta$ and a measure $\nu \in \mathfrak{M}_{+}$such as

$$
P(x, m ; B) \geq \beta \mathbf{1}_{C}(x) \nu(B), \quad B \in \mathfrak{G},
$$

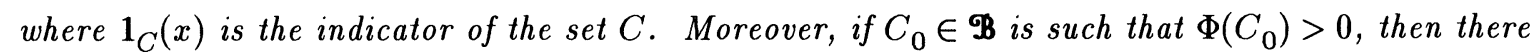
exist $C, m, \beta$ and $\nu$ such that $C \subset C_{0}$ and (1) holds.

The relation (1) is called a minorization condition and set $C$ is called a $(\nu, m)$-small set. The 
measure $\nu$ can be regarded as a probability measure, $\nu(C)=1$, without loss of generality.

Definition 4: An irreducible Markov chain $\boldsymbol{X}$ is called periodic with $d \geq 1$, if there exists a collection of subsets $\chi_{0}, \ldots, \chi_{d-1}, \chi_{i} \in \mathfrak{B}, 0 \leq i \leq d-1$, for which:

(ii) $\quad \Phi(\mathcal{N})=0$, where $\mathcal{N}=\left(\bigcup_{i=0}^{d-1} \chi_{i}\right)^{c}$

(iii) $d$ is the smallest number satisfying the conditions $(i),(i i)$.

If $d=1$, then $\boldsymbol{X}$ is called aperiodic.

Definition 5: An irreducible general chain $\boldsymbol{X}$ is Harris-recurrent if for any $C$ with $\Phi(C)>0$

$$
\mathbf{P}_{x}\left(\bigcap_{k=1}^{\infty} \bigcup_{n=k}^{\infty}\left\{X_{n} \in C\right\}\right)=1 \text { for all } x \in \chi .
$$

Proposition 3 (Orey [15]): Markov chain $X$ is Harris-recurrent if and only if there exist a subset $C \in \mathscr{B}$, an integer $m \geq 1$, a positive $\beta$, and a probability measure $\nu, \nu(C)=1$, such that both relations (1) and (2) hold.

Throughout this paper, we consider (by default) only Markov chains which are Harrisrecurrent for which condition (1) holds, even though $C, \nu, \beta$, and $m$ can differ for different chains.

Since we will use notions concerning discrete time regenerative processes, we give necessary definitions due mainly to Thorisson [18]; see also Kalashnikov [8].

Let $Z=\left(Z_{0}, Z_{1}, \ldots\right)$ be a sequence of random variables (r.v.) taking values from a complete separable metric space $\left(\mathcal{Z}, \Im_{B}\right)$ and $S=\left(S_{0}, S_{1}, \ldots\right), S_{0} \leq S_{1} \leq \ldots$, be a sequence of nonnegative integer r.v.'s. For the random pair $(Z, S)$, define the shift

$$
\theta_{n}(Z, S)=\left(\left(Z_{0}^{(n)}, Z_{1}^{(n)}, \ldots\right),\left(S_{0}^{(n)}, S_{1}^{(n)}, \ldots\right)\right)
$$

where

$$
\begin{gathered}
Z_{k}^{(n)}=Z_{k+n}, k \geq 0 \\
S_{0}^{(n)}=\min \left\{S_{i}: S_{i} \geq n, i \geq 0\right\}-n, \\
i(n)=\min \left\{i: S_{i} \geq n\right\} \\
S_{k}^{(n)}=S_{i(n)+k}-n, k \geq 0 .
\end{gathered}
$$

Definition 6: A random pair $(Z, S)$ is called a classic sense regenerative process if for any $i \geq 0$ all shifts $\theta_{S_{i}}(Z, S)$ are identically distributed and do not depend on the "prehistory" $\left(\bar{Z}_{0}, \ldots, Z_{S_{i}-1} S_{0}, \ldots, S_{i}\right)$

The sequence $S$ is called the renewal process embedded in $(Z, S)$.

Definition 7: A random pair $(Z, S)$ is called a wide sense regenerative process if for any $i \geq 0$ the shifts $\theta_{S_{i}}(Z, S)$ are identically distributed and do not depend on $\left(S_{0}, \ldots, S_{i}\right)$.

Obviously, any classic sense regenerative process is regenerative in the wide sense. Definition 7 still implies that the sequence $S$ is a renewal process which means that all inter-regeneration times $W_{i} \equiv S_{i}-S_{i-1}, i \geq 1$, are i.i.d. r.v.'s. 
Definition 8: A wide sense regenerative process $(Z, S)$ is called stationary if all shifts $\theta_{n}(Z, S), n \geq 0$, are identically distributed and hence $\theta_{n}(Z, S) \stackrel{d}{=}(Z, S)$, where $\stackrel{d}{=}$ means identity in distribution.

Definition 9: A wide sense regenerative process $\left(Z^{\prime}, S^{\prime}\right)$ is a version of a process $(Z, S)$, if

$$
\theta_{S_{0}^{\prime}}\left(Z^{\prime}, S^{\prime}\right) \stackrel{d}{=} \theta_{S_{0}}(Z, S)
$$

Proposition 4 (Thorisson [18]): In order for a stationary version $\left(Z^{\prime}, S^{\prime}\right)$ of a wide sense regenerative process $(Z, S)$ to exist it is necessary and sufficient that

$$
\boldsymbol{\mu} \equiv \boldsymbol{E}\left(S_{1}-S_{0}\right)<\infty
$$

In this case, a probability distribution of $\left(Z^{\prime}, S^{\prime}\right)$ is defined uniquely by the equality

$$
\mathbf{P}\left(\left(Z^{\prime}, S^{\prime}\right) \in \cdot\right)=\frac{1}{\mu} \boldsymbol{E}\left(\left(S_{1}-S_{0}\right) \boldsymbol{I}\left(\theta_{S_{0}}(Z, S) \in \cdot\right)\right),
$$

where $\mathbf{I}(\cdot)$ is the indicator of the event $(\cdot)$.

Consider now a splitting construction (see Nummelin [14]). In essence, it consists of embedding the initial Markov chain $\boldsymbol{X}$ into a wide sense discrete time regenerative process $(Z, S)$.

Let $\boldsymbol{X}$ be a Markov chain. Embed it into a regenerative process $(Z, S)$ which is constructed as follows. Let $Z_{n}=\left(Y_{n}, \delta_{n}\right)$, where $Y_{n}$ takes values from $\left(\chi, \Im_{B}\right)$ and $\delta_{n}$ is a binary r.v. taking values 0 and 1 . We will call $\delta_{n}$ the bell variable after Lindvall [12] (if $\delta_{n}=1$ then the bell rings). From the construction below it will follow that

$$
\boldsymbol{Y}=\left(Y_{0}, Y_{1}, \ldots\right) \stackrel{d}{=} \boldsymbol{X}
$$

Define random times

$$
\begin{gathered}
T_{0}=\min \left\{i: Y_{i} \in C\right\}, \\
T_{k+1}=\min \left\{i: Y_{i} \in C, i \geq T_{k}+m\right\}, k \geq 0 .
\end{gathered}
$$

Let $\delta_{n}, n \geq 0$, be a set of i.i.d. r.v.'s, $\mathbf{P}\left(\delta_{n}=1\right)=\beta$. Let

$$
\begin{gathered}
S_{0}=\min \left\{T_{i}+m: \delta t_{T_{i}}=1\right\}, \\
S_{k+1}=\min \left\{T_{i}+m: \delta_{T_{i}}=1, T_{i}+m>S_{k}\right\}, k \geq 0,
\end{gathered}
$$

i.e. the time is declared to be a regeneration epoch if $m$ steps ago, one of the instants (4) occurred and the bell rang that time.

If $Y_{n}=y$ and $T_{i}+m \leq n \leq T_{i+1}$ (hence, $y \notin C$ ), then we define $Y_{n+1}$ as a r.v. which depends only on $y$ and has the distribution $\mathbf{P}(y ; \cdot)$. If $Y_{n}=y$ and $n=T_{i}$ (hence, $y \in C$ ), then two cases are possible according to whether or not the bell rang at time $n$. We combine these two cases, denoting

$$
Q_{n}(\cdot)= \begin{cases}\nu(\cdot), & \text { if } \delta_{n}=1 \\ (1-\beta)^{-1}(\mathbf{P}(y, m ; \cdot)-\beta \nu(\cdot)), & \text { if } \delta_{n}=0\end{cases}
$$

Let $Y_{n+m}$ be a $r . v$. with the distribution $Q_{n}(\cdot)$ and therefore possibly dependent on $y$. Define 
the collection $Y_{n+1}, \ldots, Y_{n+m-1}$ by defining their joint distribution:

$$
\begin{aligned}
& \mathbf{P}\left(Y_{n+1} \in B_{1}, \ldots, Y_{n+m-1} \in B_{m-1} \mid Y_{n}=y, Y_{n+m}=x\right) \\
= & \mathbf{P}\left(X_{n+1} \in B_{1}, \ldots, X_{n+m-1} \in B_{m-1} \mid X_{n}=y, X_{n+m}=x\right) .
\end{aligned}
$$

Note that the probability in the right hand side of (7) is defined uniquely by the transition function of the chain $\boldsymbol{X}$.

Though the process $Z$ is not Markov in general (it is Markov under $m=1$ ), the relation (3) is satisfied. It is obvious that the constructed process $(Z, S)$ is classic sense regenerative if $m=1$ and wide sense regenerative if $m>1$. In all cases

$$
\mathbf{P}\left(Y_{S_{n}} \in \cdot\right)=\nu(\cdot), \quad n \geq 0
$$

The construction above is not unique. It is quite similar to the splitting construction suggested in Nummelin [14] for $m=1$. Other variants can be found in Kalashnikov [8] where the bell variable not only marks virtual regeneration epochs but counts residual times until these epochs in order to prove the Markov property for $Z$.

Let us agree that if we use notation $(Z, S)$, this means that it is a regenerative process constructed according to (4-7) which includes the initial Markov chain as a component.

Denote by

$$
\begin{gathered}
W_{0}=S_{0}, \\
W_{k}=S_{k}-S_{k-1}, \quad k \geq 1,
\end{gathered}
$$

successive inter-regeneration times for the process $(Z, S)$. By the construction, all $W_{k}, k \geq 1$, are i.i.d. r.v.'s. Recall that we consider here only Harris-recurrent Markov chains. Therefore, all r.v.'s $W_{k}, k \geq 0$, are finite a.s. The following two assertions are direct consequences of the construction above. Their proofs are straightforward and can be found in Kalashnikov [8].

Proposition 5: Given an irreducible aperiodic Markov chain $\boldsymbol{X}$, the r.v. $W_{1}$ is aperiodic in the sense that

$$
G C D\left\{j: \mathbf{P}\left(W_{1}=j\right)>0\right\}=1,
$$

where GCD stands for the greatest common divisor.

Let

$$
\tau_{C}=\min \left\{n: X_{n} \in C, n>0\right\}
$$

We will use the notation $c$ (possibly with indices) for different constants appearing in different relations. We will also introduce a class $\Theta$ of functions $G(n)>0, n \geq 0$, such that

$$
\Theta=\left\{G: \lim _{n \rightarrow \infty} \frac{G(n)}{n}=\infty, \lim _{n \rightarrow \infty} \frac{G(n)}{n^{2}}=0\right\}
$$

which is useful for a characterization of uniformly integrable r.v.'s; see Kalashnikov [7].

Proposition 6: For Harris-recurrent Markov chain $\boldsymbol{X}$, the following implications are true.

(i) If

$$
\int_{\chi} \mathbf{E}_{x} \tau_{C}^{s} \nu(d x) \leq c
$$


(ii)

for some $s \geq 1$, then $\mathbf{E} W_{1}^{s} \leq c_{1}=c_{1}(c, s, \beta, m)$.

If

$$
\int_{\chi} \mathbf{E}_{x} G\left(\tau_{C}\right) \nu(d x) \leq c,
$$

(iii)

$$
\begin{aligned}
& \text { for some } G \in \Theta \text {, then } \mathbf{E} G\left(W_{1}\right) \leq c_{2}=c_{2}(c, G, \beta, m) \text {. } \\
& \qquad \int_{\chi} \mathbf{E}_{x} \exp \left(\lambda \tau_{C}\right) \nu(d x) \leq c,
\end{aligned}
$$

for some $\lambda>0$, then there exists $\lambda^{\prime}(c, \lambda, \beta, m)>0$, such that

$$
\mathbf{E} \exp \left(\lambda^{\prime} W_{1}\right) \leq c_{3}=c_{3}(c, \lambda, \beta, m)
$$

The constants $c_{i}, i=1,2,3$ and $\lambda^{\prime}$ can be evaluated in a closed form in terms of the involved parameters.

For simplicity, we assume that $p_{0}(\cdot)=\nu(\cdot)$ which corresponds to the case $S_{0}=W_{0}=0$, i.e. to a zero-delayed regenerative process $(Z, S)$.

\section{Ergodic Theorems}

Let us call a Markov chain $\boldsymbol{X}^{\prime}$ a stationary version of another Markov chain $\boldsymbol{X}$ if the two chains have the same transition functions and, in addition, $\boldsymbol{X}^{\prime}$ is stationary, i.e. $X_{k}^{\prime} \stackrel{d}{=} X_{0}^{\prime}, k \geq 0$.

Theorem 1: If Markov chain $\boldsymbol{X}$ is positive recurrent, that is

$$
\int_{\chi} \mathbf{E}_{x} \tau_{C} \nu(d x)<\infty
$$

then there exists a stationary version $\boldsymbol{X}^{\prime}$.

Proof: By Proposition 6, the inequality $\mathbf{E} W_{1}<\infty$ holds for the wide sense regenerative process $(Z, S)$. In turn, this inequality is necessary and sufficient for existence a stationary version $\left(Z^{\prime}, S^{\prime}\right)$. Consider the first component $\boldsymbol{Y}^{\prime}=\left(Y_{0}^{\prime}, Y_{1}^{\prime}, \ldots\right)$ of the process $Z^{\prime}$. Evidently, it is a stationary sequence. Prove that it comprises a Markov chain. Suppose first that the chain $\boldsymbol{X}$ is aperiodic. Denote $\pi(\cdot)=\mathbf{P}\left(Y_{0}^{\prime} \in \cdot\right)$. Since $\mu<\infty$ and $W_{1}$ is an aperiodic r.v. by Proposition 5, then

$$
\operatorname{Var}\left(X_{n}, Y_{n}\right)=\operatorname{Var}\left(X_{n}, Y_{0}\right) \rightarrow 0, \text { when } n \rightarrow \infty,
$$

where Var stands for the total variation metric (see Kalashnikov [4], Thorisson [17]). Hence,

$$
\lim _{n \rightarrow \infty} \mathbf{P}(x, n ; \cdot)=\pi(\cdot)
$$

for any $x \in \chi$. It follows that

$$
\pi(\cdot)=\int_{\chi} \mathbf{P}(x ; \cdot) \pi(d x) .
$$

Introduce now a Markov chain $\boldsymbol{X}^{\prime}$ with the transition function $\mathbf{P}(x ; \cdot)$ and the initial distribution $\pi(\cdot)$. Equation $(12)$ yields that $\boldsymbol{X}^{\prime}$ is stationary. By Proposition $4, \boldsymbol{X}^{\prime} \stackrel{d}{=} \boldsymbol{Y}$, which completes the proof in the aperiodic case. The periodic case can be treated by using standard arguments, 
reducing it to an aperiodic case by considering the Markov chain each $d$ steps.

The following statement discloses the rate of convergence in the ergodic theorem.

Corollary: If Markov chain $\boldsymbol{X}$ is aperiodic and at least one of the cases ( $i$ ) (for $s>1$ ), (ii) or (iii) from Proposition 6 takes place, then

$$
\operatorname{Var}\left(X_{n}, X_{0}^{\prime}\right) \leq \frac{c}{h(n)}
$$

where:

$$
\begin{aligned}
& h(n)=s n^{s-1} \text { in the case }(i) \\
& h(n)=G(n) / n \text { in the case }(i i) \\
& h(n)=\exp (\delta n), \text { where } 0<\delta \leq \lambda^{\prime}, \text { in the case }(i i i) .
\end{aligned}
$$

The proof follows from Propositions 5 and 6 and from the rates of convergence to the steady state for regenerative processes contained in Kalashnikov [4, 7], Kalashnikov and Rachev [10], and Thorisson [17].

In order to evaluate the constant $c$ in equation (13), one can use estimates of $c_{i}$ from Proposition 6 as well as bounds of moments of the r.v. $\tau_{C}$ which can be obtained, for instance, by the test functions methods: see Kalashnikov [4, 7, 8], and Meyn and Tweedie [13].

\section{Continuity}

Very often, we can not obtain necessary characteristics of a Markov chain or we do not know exactly its transition function. In such cases we need to investigate a continuity property of Markov chains, namely, to learn whether "small deviations" of transition functions lead to "small deviations" of non-stationary or stationary distributions of the chains. To this end, we introduce a notion Feller chain. Let $f: \boldsymbol{\chi} \rightarrow \boldsymbol{R}^{1}$ be a real function defined on the state space of a chain $\boldsymbol{X}$. Define the operator

$$
\mathbf{P} f(x):=\int_{\chi} f(y) \mathbf{P}(x ; d y)
$$

when the integral in the right hand side of (14) converges.

Definition 10 (Meyn and Tweedie [13]): Markov chain $\boldsymbol{X}$ is called a weak Feller chain if the function $\mathbf{P} f(x)$ is continuous and bounded provided that $f$ is continuous and bounded. If the mapping $P f$ is continuous and bounded for any measurable bounded function $f$, then $\boldsymbol{X}$ is called a strong Feller chain.

The following assertion is trite but we will need this in the sequel.

Lemma 1: Let $\boldsymbol{X}^{(n)}, n \geq 0$, be a sequence of weak Feller chains with the transition functions $\mathbf{P}^{(n)}$. If

$$
\mathbf{P}^{(n)} f(x) \rightarrow \mathbf{P}^{(0)} f(x) \quad \forall x \in \chi,
$$

for any continuous and bounded $f$, then

$$
\int_{\chi} f(y) \mathbf{P}^{(n)}(x, k ; d y) \rightarrow \int_{\chi} f(y) \mathbf{P}^{(0)}(x, k ; d y) \quad \forall x \in \chi,
$$

for any fixed $k>0$.

If $\boldsymbol{X}^{(n)}, n \geq 0$, is a sequence of strong Feller chains, then the statement above holds for any 
bounded measurable function $f$.

We start with the case when all chains $\boldsymbol{X}^{(n)}, n \geq 0$, are strong Feller and $\boldsymbol{X}^{(0)}$ satisfies the minorization condition

$$
\mathbf{P}^{(0)}(x, m ; B) \geq \beta^{\prime} 1_{C}(x) \nu^{\prime}(B), \quad B \in \mathscr{\Im}
$$

By the strong Feller property, there exist $\beta$ and $\nu$ such that

$$
\mathbf{P}^{(n)}(x, m ; B) \geq \beta \mathbf{1}_{C}(x) \nu(B), B \in \mathscr{B}
$$

for all $n$ beginning from some $n_{0} \geq 0$. Because we are interested in the case $n \rightarrow \infty$, we can put $n_{0}=0$ without loss of generality.

Let us embed the chains $\boldsymbol{X}^{(n)}$ into regenerative processes $\left(Z^{(n)}, S^{(n)}\right), n \geq 0$, constructed with the aid of condition (18) and assume, as earlier, that the instant zero is a simultaneous regeneration epoch for all those processes. In particular, all $X_{0}^{(n)}, n \geq 0$, have the same distribution $\nu$. Denote by $\left(W_{k}^{(n)}\right)_{k \geq 1}$, successive inter-regeneration times for the $n$-th process and set for $n \geq 0$

$$
\begin{gathered}
\tau_{C}^{(n)}=\min \left\{k: X_{k}^{(n)} \in C, k>0\right\}, \\
b_{k}^{(n)}=\int_{\chi} \mathbf{P}\left(\tau_{C}^{(n)}=k \mid X_{0}^{(n)}=y\right) \nu(d y), k \geq 1 .
\end{gathered}
$$

Theorem 2: Let all chains $\boldsymbol{X}^{(n)}, n \geq 0$, be strong Feller satisfying the common minorization condition (18), and $\mathbf{P}^{(n)} f(x) \rightarrow \mathbf{P}^{(0)} f(x)$ for any bounded $f$ and any $x \in \chi$. Let, in addition, the chain $\boldsymbol{X}^{(0)}$ be irreducible and aperiodic and the family of distributions $\left(b_{k}^{(n)}\right)_{k}>0, n \geq 0$, be uniformly integrable. Then

$$
\lim _{n \rightarrow \infty} \sup _{k \geq 0} \operatorname{Var}\left(X_{k}^{(n)}, X_{k}^{(0)}\right)=0
$$

Proof: By the strong Feller property,

$$
\lim _{n \rightarrow \infty} b_{k}^{(n)}=b_{k}^{(0)}
$$

for any fixed $k>0$. Therefore, without loss of generality, all chains $\boldsymbol{X}^{(n)}$ can be regarded as aperiodic and irreducible. Moreover, the distributions $\left(b_{k}^{(n)}\right)_{k \geq 0}, n \geq 0$, are uniformly aperiodic in the sense that there exist integer $N$ and real $\alpha>0$ such that

$$
G C D\left\{k: b_{k}^{(n)} \geq \alpha, \quad 1 \leq k \leq N\right\}=1
$$

for all $n \geq 0$. Since the family $\left(b_{k}^{(n)}\right)_{k}>0, n \geq 0$, is uniformly integrable, there exists a function $G \in \Theta$ and a constant $g$ such that

$$
\mathbf{E} G\left(\tau_{C}^{(n)}\right) \leq g
$$

for all $n \geq 0$ (see Kalashnikov [4]). It follows from Proposition 6(ii), that

$$
\sup _{n} \mathbf{E} G\left(W_{1}^{(n)}\right) \leq c<\infty
$$

By Lemma 1 and construction of the regenerative processes $\left(Z^{(n)}, S^{(n)}\right)$, 


$$
\lim _{n \rightarrow \infty} \operatorname{Var}\left(Z_{k}^{(n)}, Z_{k}^{(0)}\right)=\lim _{n \rightarrow \infty} \operatorname{Var}\left(X_{k}^{(n)}, X_{k}^{(0)}\right)=0
$$

for any $k \geq 0$. According to Theorem 6.5.3 from Kalashnikov [4] (see also Corollary 1 to Theorem 3.5.1 in Kalashnikov [8]), the inequality (20) is a consequence of relations (24) and (25) $\square$

What is more, it is possible to obtain a quantitative bound on

$$
\sup _{k} \operatorname{Var}\left(X_{k}^{(n)}, X_{k}^{(0)}\right)
$$

given that one can estimate

$$
\epsilon(k)=\max _{j \leq k} \operatorname{Var}\left(X_{j}^{(n)}, X_{j}^{(0)}\right)
$$

see Kalashnikov [8].

The requirement that $\boldsymbol{X}^{(n)}$ are strong Feller chains is restrictive enough (though, instead we get a continuity property in terms of the total variation metric). We now relax this, requiring that all $\boldsymbol{X}^{(n)}$ are weak Feller chains. In this situation, the relation (17) does not yield (18), in general, and limiting relation (21) can be violated. So, additional restrictions, appearing in the following Theorem 3 , are engaged to overcome undesirable consequences of such violations. In addition, we will need a "weak" metric $\zeta_{B L}$ for a metrization of a weak convergence of $\boldsymbol{X}^{(n)}$ to $\boldsymbol{X}^{(0)}$ which is defined as follows (see Kalashnikov and Rachev [10]):

$$
\zeta_{B L}(X, Y)=\sup \{|\mathbf{E} f(X)-\mathbf{E} f(Y)|:|f| \leq 1,|f(x)-f(y)| \leq h(x, y)\}
$$

where $f: \boldsymbol{\chi} \rightarrow \boldsymbol{R}^{1}, h$ is a metric in the space $\boldsymbol{\chi}, X$ and $Y$ are $r . v$.'s with values from the space $\boldsymbol{\chi}$.

Theorem 3: Let all $\boldsymbol{X}^{(n)}, n \geq 0$, be weak Feller chains satisfying the "common" minorization condition (18) and $\mathbf{P}^{(n)} f(x) \rightarrow \mathbf{P}^{(0)} f(x)$ for any bounded continuous $f$ and any $x \in \boldsymbol{\chi}$. Let, in addition, chain $\boldsymbol{X}^{(0)}$ is irreducible and aperiodic, and the family of distributions $\left(b_{k}^{(n)}\right)_{k \geq 1}, n \geq 0$, is uniformly integrable and uniformly aperiodic, i.e. the relation (22) holds. Then

$$
\lim _{n \rightarrow \infty} \sup _{k \geq 0} \zeta_{B L}\left(X_{k}^{(n)}, X_{k}^{(0)}\right)=0
$$

The proof completely repeats that of Theorem 2 .

Statements of Theorems 2 and 3 admit a quantification which follows from results obtained in Kalashnikov [4] and [8]. Display only a bound which is valid under a "power case" $G(n)=n^{s}$, $s>1$. let probability metric $d$ is defined as $d=V a r$ in the case of Theorem 2 and $d=\zeta_{B L}$ in the case of Theorem 3 . Then, in both cases,

$$
\sup _{k} d\left(X_{k}^{(n)}, X_{k}^{(0)}\right) \leq \inf _{T}\left\{\max _{k \leq T} d\left(X_{k}^{(n)}, X_{k}^{(0)}\right)+\frac{c}{T^{s-1}}\right\}
$$

In particular, if

$$
\max _{k \leq T} d\left(X_{k}^{(n)}, X_{k}^{(0)}\right) \leq \epsilon(n) T
$$

then

$$
\sup _{k}\left(X_{k}^{(n)}, X_{k}^{(0)}\right) \leq c(\epsilon(n))^{(s-1) / s}
$$

Relation (27) arise naturally in queueing theory; see Kalashnikov [7]. For example, single-server, multi-server and multi-phase models can be described by general Markov chains satisfying finitetime continuity inequality (27). 


\section{Approximations}

Let us turn to a problem which is complementary to that considered in Section 4 . Specifically, we now consider an approximation problem which can be outlined as follows. Let $\boldsymbol{X}=\boldsymbol{X}^{(0)}$ be a general Markov chain. The problem consists of the construction of a sequence of $\boldsymbol{X}^{(n)}$ belonging to a prescribed class (e.g., having a finite number of states) such that (26) is true.

First, let us assume that the chain $\boldsymbol{X}^{(0)}$ with the transition probabilities $\mathbf{P}^{(0)} \equiv \mathbf{P}(x ; \cdot)$ satisfies the minorization condition (1). In addition, let $\Gamma_{n}, n \geq 0$, be a collection of nested compacts such that

$$
C \subset \Gamma_{0} \subset \Gamma_{1} \subset \Gamma_{2} \subset \ldots ; \quad \bigcup_{n} \Gamma_{n}=\chi
$$

For each $n \geq 1$, define the chain $\boldsymbol{X}^{(n)}$ as a restriction of the chain $\boldsymbol{X}^{(0)}$ to the compact $\Gamma_{n}$. It has the following transition probabilities:

$$
\mathbf{P}^{(n)}(x ; B)=\mathbf{P}(x ; B)+\mathbf{P}\left(x ; \chi \backslash \Gamma_{n}\right) \nu(B), \quad B \subset \Gamma_{n} .
$$

Let $W_{k}^{(n)}, k \geq 1$, be inter-regeneration times for the process $\left(Z^{(n)}, S^{(n)}\right), n \geq 1$, into which $\boldsymbol{X}^{(n)}$ is embedded. Denote $W_{k} \equiv W_{k}^{(0)}$. By (28),

$$
\mathbf{P}\left(W_{1} \leq x\right) \leq \mathbf{P}\left(W_{1}^{(n)} \leq x\right)
$$

for all $x \geq 0$ and $n \geq 1$. In particular, $\mathbf{E} W_{1} \geq \mathbf{E} W_{1}^{(n)}$. Hence, the collection of r.v.'s $\left(W_{1}^{(n)}\right)_{n \geq 0}$ forms a uniformly integrable family.

Lemma 2: Let $\boldsymbol{X}$ be an aperiodic strong Feller chain satisfying the minorization condition (1), and assume the processes $\boldsymbol{X}^{(n)}, n \geq 1$, are constructed according to (28). Then

$$
\lim _{n \rightarrow \infty} \sup _{k \geq 0} \operatorname{Var}\left(X_{k}, X_{k}^{(n)}\right)=0 .
$$

The proof immediately follows from the fact that $\boldsymbol{X}^{(n)}$ are strong Feller chains, Lemma 1 and Theorem 2.

Similarly, using Theorem 3, one can prove the following.

Lemma 3: Let $\boldsymbol{X}$ be an aperiodic weak Feller chain satisfying the minorization condition (1), and the processes $\boldsymbol{X}^{(n)}, n \geq 1$, are constructed according to (28). Then

$$
\lim _{n \rightarrow \infty} \sup _{k \geq 0} \zeta_{B L}\left(X_{k}, X_{k}^{(n)}\right)=0 .
$$

Without loss of generality, we can (and will) assume that, in each subset $\Gamma_{n}$, the mean residual time until the nearest regeneration time of the process $\left(Z^{(n)}, S^{(n)}\right)$ is bounded from above by $n$ given $\mathbf{E} W_{1}<\infty$. Really, if $\mathbf{E} W_{1}<\infty$, then $\mathbf{E} W_{1}^{(n)} \leq \mathbf{E} W_{1}<\infty$ and one can redefine (if necessary):

$$
\Gamma_{n}:=\Gamma_{n} \cap\left\{x: R_{x}^{(n)} \leq n\right\}
$$

where $R_{x}^{(n)}$ is a forward recurrence time for the renewal process $S^{(n)}$ given that $X^{(n)}$ starts from the state $x$.

Lemma 2 and 3 show how to approximate a general Markov chain $\boldsymbol{X}^{(0)}$ by another chain $\boldsymbol{X}^{(n)}$ having a compact state space. We now move on to conditions ensuring the possibility of approximating Markov chain $\boldsymbol{X}^{(n)}$ with a compact state space $\Gamma_{n}$ by a finite chain. 
Take an arbitrary $\epsilon>0$ which can be treated as the accuracy of approximation. Let $\alpha_{0}, \ldots, \alpha_{N}$ be a $\epsilon$-net in $\Gamma_{n}$ such that $\alpha_{0} \in C$ (evidently, $N$ may depend on $\epsilon$ ). Divide $\Gamma_{n}$ into $N+1$ subsets $\Gamma_{n}^{(0)}, \ldots, \Gamma_{n}^{(N)}$ as follows:

$$
\begin{aligned}
\Gamma_{n}^{(0)}= & \left\{x: h\left(x, \alpha_{0}\right) \leq \epsilon, x \in \Gamma_{n}\right\} \\
\Gamma_{n}^{(1)}= & \left\{x: h\left(x, \alpha_{1}\right) \leq \epsilon, x \in \Gamma_{n} \backslash \Gamma_{n}^{(0)}\right\} \\
\Gamma_{n}^{(2)}= & \left\{x: h\left(x, \alpha_{2}\right) \leq \epsilon, x \in \Gamma_{n} \backslash\left(\Gamma_{n}^{(0)} \cup \Gamma_{n}^{(1)}\right)\right\} \\
& \vdots \\
\Gamma_{n}^{(N)}= & \left\{x: h\left(x, \alpha_{N}\right) \leq \epsilon, x \in \Gamma_{n} \backslash\left(\Gamma_{n}^{(0)} \cup \ldots \cup \Gamma_{n}^{(N-1)}\right)\right\} .
\end{aligned}
$$

Recall that $h$ stands for the metric that the complete separable metric space $\chi$ is equipped with. Each $\Gamma_{n}^{(j)}$ represents a neighborhood of the point $\alpha_{j}$, its radius being less than or equal to $\epsilon$. Let

$$
p_{i j}^{(\epsilon)}=\mathbf{P}^{(n)}\left(\alpha_{i} ; \Gamma_{n}^{(j)}\right), \quad i, j=0, \ldots, N
$$

and introduce a finite Markov chain $\boldsymbol{X}^{(n, \epsilon)}$ with state space $\left\{\alpha_{0}, \ldots, \alpha_{N}\right\}$, transition probabilities (38), and initial state $X_{0}^{(n, \epsilon)}=\alpha_{j}$ if $X_{0}^{(n)} \in \Gamma_{n}^{(j)}$. In order to compare $\boldsymbol{X}^{(n, \epsilon)}$ with $\boldsymbol{X}^{(n)}$, we embed $\boldsymbol{X}^{(n, \epsilon)}$ into a regenerative process $\left(Z^{(n, \epsilon)}, S^{(n, \epsilon)}\right)$ in a quite similar way as it has been done for the process $X^{(n)}$. Denote inter-regeneration times for $\left(Z^{(n, \epsilon)}, S^{(n, \epsilon)}\right)$ by $\left(W_{k}^{(n, \epsilon)}\right)_{k \geq 1}$.

Lemma 4: Let $\boldsymbol{X}$ satisfy the conditions of Lemma 2. Suppose that $\boldsymbol{X}^{(n)}$ is a Markov chain constructed as in (28). Then, for any fixed $n$,

$$
\limsup _{\epsilon \rightarrow 0} \operatorname{su}_{B L}\left(X_{k}^{(n)}, X_{k}^{(n, \epsilon)}\right)=0
$$

Proof: Let $\tau_{C}^{(n)}=\min \left\{k: k>0, X_{k}^{(n)} \in C\right\}$ and define a test function (see Kalashnikov [4, 7, 8], and Meyn and Tweedie [13]) by the equation

$$
V(x)=\mathbf{E}_{x} \tau_{C}^{(n)}
$$

By remark to Lemma 3 , we can assume (without loss of generality) that

$$
\sup _{x \in \Gamma_{n}} V(x) \leq n .
$$

Let $\mathbf{A}^{(n)}$ be a generating operator of the Markov chain $\boldsymbol{X}^{(n)}$. Then equation (34) yields

$$
\mathbf{A}^{(n)} V(x)=-1, \quad x \notin C .
$$

By the strong Feller property of $\boldsymbol{X}$, the chain $\boldsymbol{X}^{(n)}$ is strong Feller too, and, hence, $V(x)$ is continuous in $\Gamma_{n}$. Therefore,

$$
\sup _{1 \leq j \leq N} \mathbf{A}^{(n, \epsilon)} V\left(\alpha_{j}\right) \leq-\Delta(\epsilon)<0
$$

where $\mathbf{A}^{(n, \epsilon)}$ is the generating operator of the finite chain $\boldsymbol{X}^{(n, \epsilon)}$ and $\Delta(\epsilon) \rightarrow 1$ as $\epsilon \rightarrow 0$. Function $V(x)$ is bounded in $\Gamma_{n}$; see (35). This fact, together with relations (36) and (37), yields that all conditions of Corollary 3 to Theorem 1.1.5 in Kalashnikov [8] (see also Kalashnikov and Rachev [10], Theorem 2, Appendix 5) hold, which, in turn, imply that 


$$
\sup \left\{\mathbf{E}_{x}\left(\tau_{C}^{(n, \epsilon)}\right)^{s}: x \in C, \epsilon>0\right\} \leq c<\infty
$$

for any $s>1$ (for example, for $s=2$ ). Therefore, by Proposition 5, the family of r.v.'s $\left(W_{1}^{(n, \epsilon)}\right)_{\epsilon>0}$ is uniformly integrable. The strong Feller property of $\boldsymbol{X}^{(n)}$ and the construction (32) yield

$$
\lim _{\epsilon \rightarrow \infty} \zeta_{B L}\left(X_{k}^{(n)}, X_{k}^{(n, \epsilon)}\right)=0
$$

for any fixed $k$, where $k=0$ is a simultaneous regeneration time for both $\boldsymbol{X}^{(n)}$ and $\boldsymbol{X}^{(n, \epsilon)}$. This means the relation (33) is a consequence of Theorem 4.

Theorem 4: Let $\boldsymbol{X}$ be an aperiodic Harris-recurrent, positive, strong Feller chain. Then there exists a sequence of finite chains $\boldsymbol{X}(N)$ such that

$$
\lim _{N \rightarrow \infty} \sup _{n} \zeta_{B L}\left(X_{n}, X_{n}(N)\right)=0
$$

The proof follows from statements of Lemmas 2 and 4 if one takes $\boldsymbol{X}(N)=\boldsymbol{X}^{(n, \epsilon)}$.

Having examined the proof of Lemma 4, we can notice that the strong Feller property is required only for the continuity of $V(x)$. But the proof is still valid if one supposes that there exists a bounded continuous function $V(x)$ such that

$$
\mathbf{A} V(x) \leq-\Delta<0, x \notin C .
$$

Therefore, using Lemma 3 and repeating main arguments of the proof of Theorem 4, we arrive at a weaker version of the above statement.

Theorem 5: Let $\boldsymbol{X}$ be an aperiodic Harris-recurrent, positive, weak Feller chain, and let there exist a continuous function $V(x)$ such that relation (40) holds. Then there exists a sequence of finite chains $\boldsymbol{X}(N)$ such that the relation (45) is still true.

Let us mark the following two circumstances. First, for many queuing models (single- and multi-server, multi-phase and others) test functions $V(x)$ satisfying Theorem 5 have been known; see Kalashnikov [7, 8]. Second, the limiting relation (39) can be quantified just like it has been for the continuity problem in Section 4 .

\section{Rarity and Exponentiality}

Suppose now that general Markov chain $\boldsymbol{X}$ visits some subset $Q \subset \boldsymbol{\chi}$ infrequently. Then it can be expected that the passage time from some specific initial state (random, in general) to $Q$ is exponentially distributed to good approximation. Similar problems have been investigated earlier, mainly, by analytic tools; see Keilson [11]. We will now show how to solve them by probabilistic methods.

Let us suppose that $\boldsymbol{X}$ satisfies (1) with $m=1$. It follows that, in this case, $\boldsymbol{X}$ can be embedded into a classic sense regenerative process $(Z, S)$. But we would like to emphasize that the restriction $m=1$ is not of exceptional importance and can be relaxed. Suppose, in addition, that $(Z, S)$ is a zero-delayed process, i.e. $S_{0}=0$, which implies that the initial distribution of $\boldsymbol{X}$ coincides with $\nu(B), B \in \Re$. Formalize the supposition about "rare visits" to $Q$ as follows. Let $\Gamma_{j}, j \geq 0$, be a sequence of nested subsets of $\chi$ such that

$$
\Gamma_{j} \subset \Gamma_{j+1}, \quad j \geq 0, \bigcup_{j=0}^{\infty} \Gamma_{j}=\chi .
$$


Define the first passage time of the subset $Q=Q_{j}=\chi \backslash \Gamma_{j}$ as

$$
\tau_{j}=\min \left\{k: X_{k} \notin \Gamma_{j}, \quad k \geq 0\right\}
$$

Denote bo $\mathbf{P}_{\nu}$ and $\mathbf{E}_{\nu}$ the conditional probability and expectation provided that $X_{0}$ is a r.v. having the distribution $\nu$.

In order to state the results, we need some propositions associated with so-called "geometric sums" of i.i.d. r.v.'s. Let $\boldsymbol{Y}=\left(Y_{i}\right)_{i>1}$ be a sequence of nonnegative i.i.d. r.v.'s with a finite mean $\mu$, and let $Y_{0}$ be a r.v. which does not depend on the sequence $\boldsymbol{Y}$. Suppose that $\kappa$ is an integer r.v. that does not depend on both $Y$ and $Y_{0}$ and has a geometric distribution

Let

$$
\mathbf{P}(\kappa=k)=q(1-q)^{k-1}, \quad k \geq 1,0<q<1 .
$$

$$
\boldsymbol{B}(x)=\mathbf{P}\left(Y_{1} \leq x\right), \boldsymbol{B}_{0}(x)=\mathbf{P}\left(Y_{0} \leq x\right) .
$$

Consider two random sums

$$
\begin{gathered}
\sigma=Y_{1}+\ldots+Y_{\kappa} \\
\sigma_{0}=Y_{1}+\ldots+Y_{\kappa-1}+Y_{0}
\end{gathered}
$$

and denote their distribution functions by

$$
\boldsymbol{W}(x)=\mathbf{P}(\sigma \leq x), \quad \boldsymbol{W}_{0}(x)=\mathbf{P}\left(\sigma_{0} \leq x\right) .
$$

From (45) and (46), we have

$$
\boldsymbol{W}_{0}(x)=(1-q) \boldsymbol{W} * \boldsymbol{B}_{0}(x)+q \boldsymbol{B}_{0}(x) .
$$

Relation (48) shows that if the distribution functions $\boldsymbol{B}$ and $\boldsymbol{B}_{0}$ vary along $q \rightarrow 0$ in such a way that $\boldsymbol{B}_{0}(x) \rightarrow 1$ for any fixed $x$ and the distribution function $\boldsymbol{W}$ has some limit then the distribution function $\boldsymbol{W}_{0}$ has the same limit.

Let us find conditions ensuring that a $r . v . \sigma$ after "normalization" has a limiting exponential distribution function. These conditions generalize the conditions of a well-known theorem of Renyi; see Daley and Vere-Jones [2]. Define, for each $x>0$,

$$
\boldsymbol{M}_{1}(x)=\int_{0}^{x}(1-\boldsymbol{B}(u)) d u .
$$

Proposition 7: Let the distribution function $\boldsymbol{B}$ vary in such a way that for each fixed $x>0$

$$
\lim _{q \rightarrow 0} \frac{1}{\mu} \boldsymbol{M}_{1}\left(\frac{\mu x}{q}\right)=1
$$

Then

$$
\lim _{q \rightarrow 0} \boldsymbol{W}\left(\frac{\mu x}{q}\right)=1-\exp (-x)
$$

The proof of Proposition 7 can be found in Kalashnikov [8] (Theorem 4.2.1), where one can also find two-sided bounds of the distribution function $\boldsymbol{W}$. Similar estimates under an assumption that there exists the second moment for the r.v. $Y_{1}$ are obtained in Kalashnikov [6]. Numerous properties of geometric sums are considered in Kalashnikov [9]. Proposition 7 and equation (48) yield the following statement.

Corollary: If, under assumptions of Proposition 7, the distribution function $\boldsymbol{B}_{0}$ varies in such 
a way that

$$
\lim _{q \rightarrow 0} B_{0}\left(\frac{\mu x}{q}\right)=1
$$

for any fixed $x>0$, then

$$
\lim _{q \rightarrow 0} \boldsymbol{W}_{0}\left(\frac{\mu x}{q}\right)=1-\exp (-x)
$$

Now, return to the chain $\boldsymbol{X}$.

Theorem 6: Let $\boldsymbol{X}$ satisfy assumptions imposed above in this section and

$$
\begin{gathered}
\mathbf{E}_{\nu} \tau_{j}=\mu_{j} \rightarrow \infty \text {, when } j \rightarrow \infty \\
\mathbf{E}_{\nu} \tau_{C}<\infty
\end{gathered}
$$

Then, for any $x \geq 0$,

$$
\mathbf{P}_{\nu}\left(\frac{\tau_{j}}{\mu_{j}} \leq x\right) \rightarrow 1-\exp (-x), \text { when } j \rightarrow \infty
$$

Proof: Because of Proposition 6(i) and (51), the inter-regeneration time of $(Z, S)$ has a finite expectation

$$
\mathbf{E} W_{1}=\mu^{0}<\infty
$$

It follows from (50) and (51) that

$$
\begin{gathered}
q=\mathbf{P}_{\nu}\left(\tau_{j}<W_{1}\right) \rightarrow 0, \\
\mathbf{E}_{n}\left(\tau_{j} ; \tau_{j}<W_{1}\right) \rightarrow 0, \\
\mathbf{E}_{\nu}\left(W_{1} \mid W_{1} \leq \tau_{j}\right)=\mu \rightarrow \mu^{0},
\end{gathered}
$$

when $j \rightarrow \infty$.

Now let

$$
\begin{gathered}
\boldsymbol{B}(x)=\mathbf{P}_{\nu}\left(W_{1} \leq x \mid W_{1} \leq \tau_{j}\right) \\
\boldsymbol{B}_{0}(x)=\mathbf{P}_{\nu}\left(\tau_{j} \leq x \mid W_{1}>\tau_{j}\right) .
\end{gathered}
$$

Then, by the fact that $(Z, S)$ is a classic sense regenerative process, r.v. $\tau_{j}$ defined by (42) has the same distribution $\boldsymbol{W}_{0}$ as the r.v. $\sigma_{0}$ defined by (46). In particular, according to (54)-(56), we arrive at the relation

$$
\lim _{j \rightarrow \infty} \frac{q \mu_{j}}{\mu}=\lim _{j \rightarrow \infty} \frac{q \mu_{j}}{\mu^{0}}=1 .
$$

Relations (54) and (56) imply also that all assumptions of Proposition 7 are fulfilled. In addition, (54) and (55) yield that

$$
\lim _{j \rightarrow \infty} q \mathbf{E}_{\nu}\left(\tau_{j} \mid \tau_{j}<W_{1}\right)=0
$$

and so $\lim _{q \rightarrow 0} \boldsymbol{B}_{0}(\mu x / q)=1$ for any $x \geq 0$. Therefore, corollary of Proposition 7 yields (52).

As we have noted, convergence rate estimates in Theorem 6 can be obtained with the help of 
the results contained in Kalashnikov [6,8,9]. The assertion of Theorem 6 was proved in Keilson

[11] (Theorem $8.2 \mathrm{~B}$ ) by analytic methods.

\section{References}

[1] Asmussen, S., Applied Probability and Queues, J. Wiley \& Sons, Chichester 1987.

[2] Daley, D., and Vere-Jones, D., An Introduction to the Theory of Point Processes, Springer-Verlag, New York 1988.

[3] Feller, W., An Introduction to Probability Theory and Its Applications Vol. 1, Third Edition, J. Wiley \& Sons, New York 1968.

[4] Kalashnikov, V., Qualitative Analysis of the Behavior of Complex Systems by the Method of Test Functions, Nauka, Moscow (in Russian) 1978.

[5] Kalashnikov, V., Crossing and comparison of regenerative processes, Acta Appl. Math., 34, (1994), 151-172.

[6] Kalashnikov, V., Two-side estimates of geometric convolutions, In: Lecture Notes in Math., 1546 (1993), 76-88.

[7] Kalashnikov, V., Mathematical Methods in Queueing Theory, Kluwer Acad. Publ., Dordrecht 1994.

[8] Kalashnikov, V., Topics in Regenerative Processes, CRC Press, Boca Raton, Florida 1994.

[9] Kalashnikov, V., Geometric Sums and Their Applications, Chapman and Hall, New York (in preparation) 1995.

[10] Kalashnikov, V., and Rachev, S., Mathematical Methods for Construction of Queueing Models, Wadsworth \& Brooks/Cole, Pacific Grove, California 1990.

[11] Keilson, J., Markov Chain Models - Rarity and Exponentiality, Springer-Verlag, New York 1979.

[12] Lindvall, T., Lectures on the Coupling Method, J. Wiley \& Sons, New York 1992.

[13] Meyn, S., and Tweedie, R., Markov Chains and Stochastic Stability, Springer-Verlag, New York 1993.

[14] Nummelin, E., General Irreducible Markov Chains and Non-Negative Operators, Cambridge University Press, Cambridge 1984.

[15] Orey, S., Lecture Notes on Limit Theorems for Markov Chain Transition Probabilities, Van Nostrand-Reinhold, London 1971.

[16] Smith, W., Renewal theory and its ramifications, J. Roy. Stat. Soc. B 20 (1958), 243-302.

[17] Thorisson, H., The coupling of regenerative processes, Adv. Appl. Prob. 15 (1983), 531-561.

[18] Thorisson, H., Construction of a stationary regenerative process, Stoch. Proc. Appl. 42 (1992), 237-253.

[19] Thorisson, H., On Time- and Cycle-Stationarity, Preprint RH-14-92, Science Institute, Univ. of Iceland 1992.

[20] Thorisson, H., Coupling and convergence of random elements, processes and regenerative processes, Acta Appl. Math. 34 (1994), 70-85. 


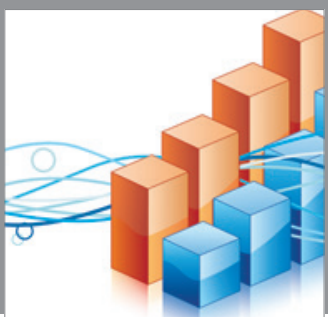

Advances in

Operations Research

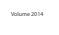

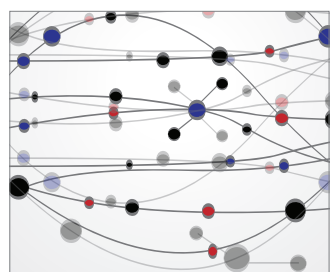

\section{The Scientific} World Journal
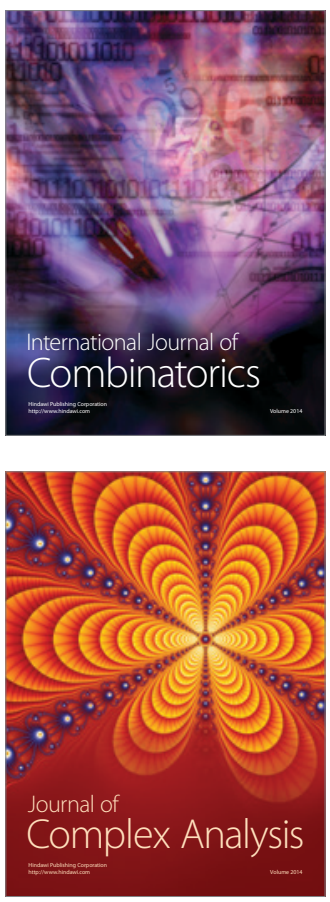

International Journal of

Mathematics and

Mathematical

Sciences
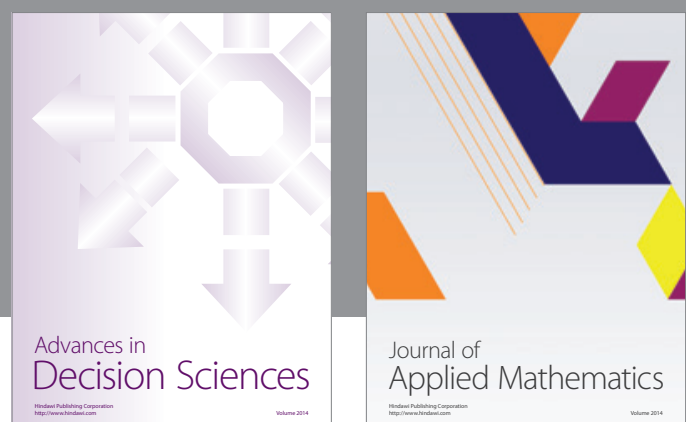

Journal of

Applied Mathematics
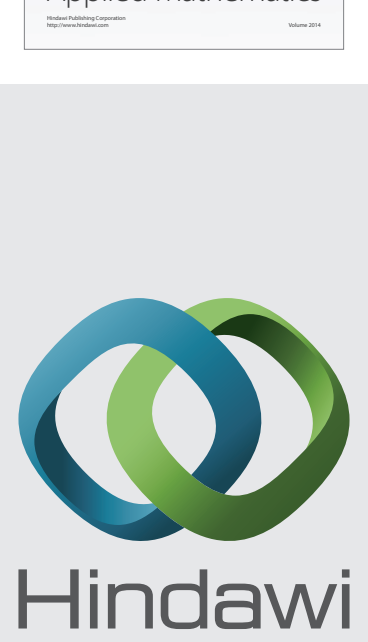

Submit your manuscripts at http://www.hindawi.com
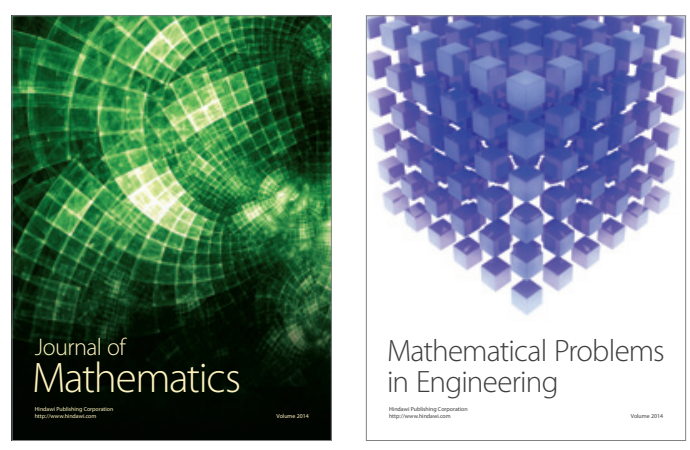

Mathematical Problems in Engineering
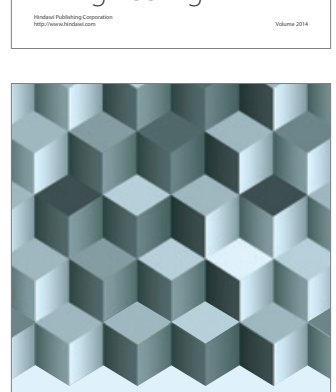

Journal of

Function Spaces
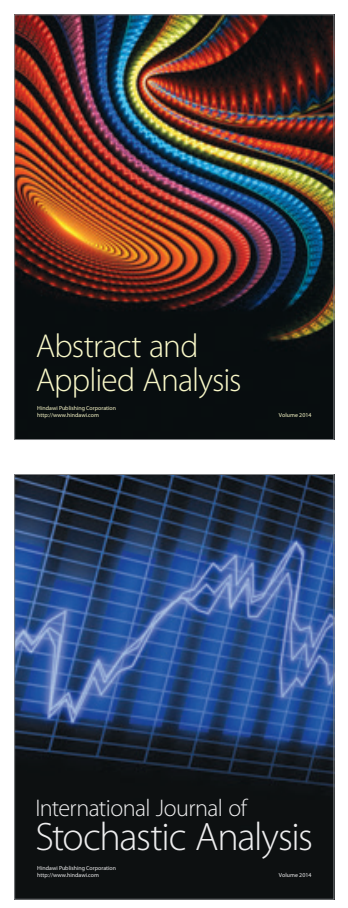

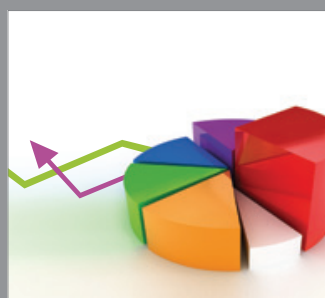

ournal of

Probability and Statistics

Promensencen
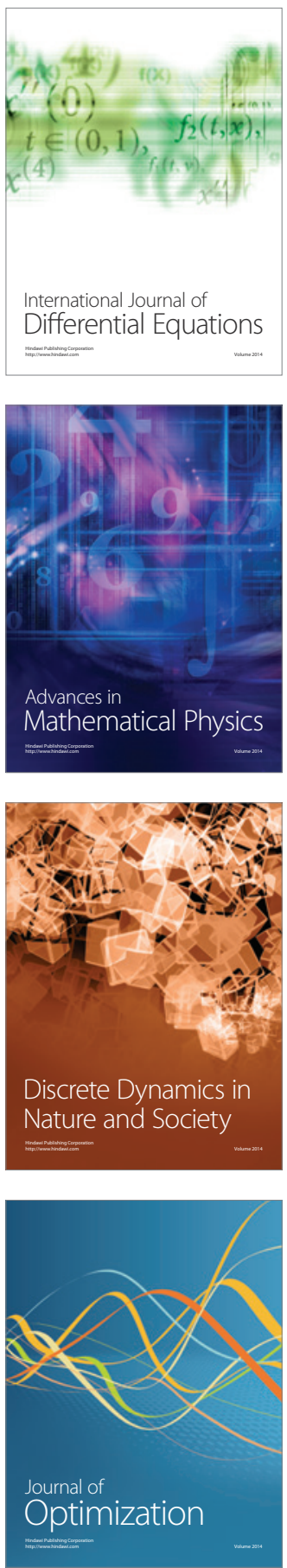